\title{
A simple mathematical model of cell clustering by chemotaxis.
}

\author{
Paul J. Harris
}

October 18, 2017

\begin{abstract}
Chemotaxis is the process by which cells and clusters of cells follow chemical signals in order to combine and form larger clusters. The spreading of the chemical signal from any given cell can be modelled using the linear diffusion equation, and the standard equations of motion can be used to determine how a cell, or cluster of cells, moves in response to the chemical signal. The resulting differential equations for the cell locations are integrated through time using the fourth-order Runge-Kutta method. The effect which changing the initial concentration magnitude, diffusion constant and velocity damping parameter has on the shape of the final clusters of cells is investigated and discussed.
\end{abstract}

\section{Introduction}

In experimental work it has been observed that cells and clusters of cells, either in vivo or in vitro, can move using the process of chemotaxis where they sense the direction in which the concentration of a chemical is increasing and move towards the region where the concentration is greatest. A number of papers have been published which illustrate experiments where chemotaxis has been observed (see $[8,16,17]$ for example).

Aggregation of cells, either singularly or in small clusters, into large clusters has been reported in the literature. For example, Jia et. al [9] report on the aggregation and self-assembly of clusters of rodent pancreatic cells, and Hilderink et. al. [7] report on a study into the aggregation of human pancreatic cells into clusters suitable for transplantation. An example of the formation of clusters of pancreatic cells is shown in Figure 1 (obtained, with thanks, from The Brighton Centre for Regenerative Medicine, University of Brighton). Here each left-hand sub-figure is a frame taken from a time-lapse video of an experiment to observe the formation of clusters, and the right-hand figures shows the circles that are used to represent the locations of the individual cells in the type of mathematical model that is being proposed in this paper. The times of the frames are given on a linear scale but are otherwise in arbitrary units. The frames from the video show how the cells combine together to form clusters over time. In particular, the video shows how a large number of individual cells can combine together to form a large cluster.

The aim of this paper is to develop a simple mathematical model of how the cells combine together to form clusters, as observed in Figure 1. The model 

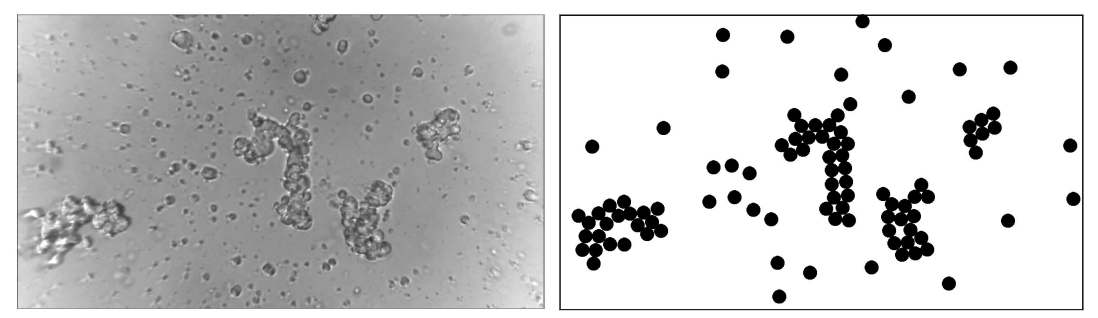

(a) $t=0$

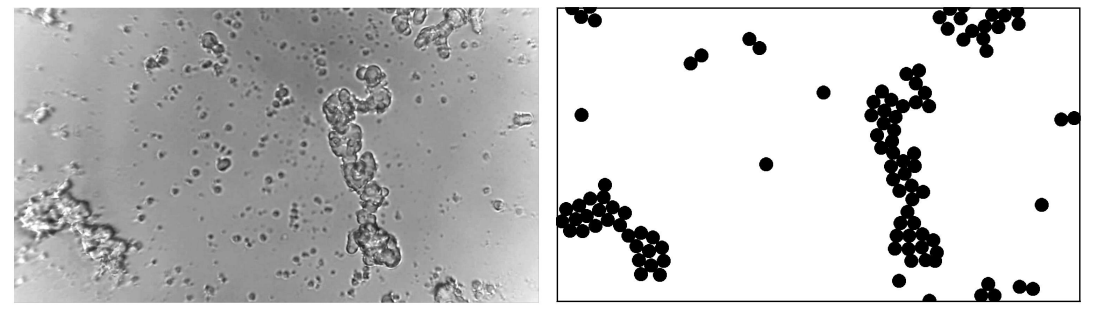

(b) $t=2$

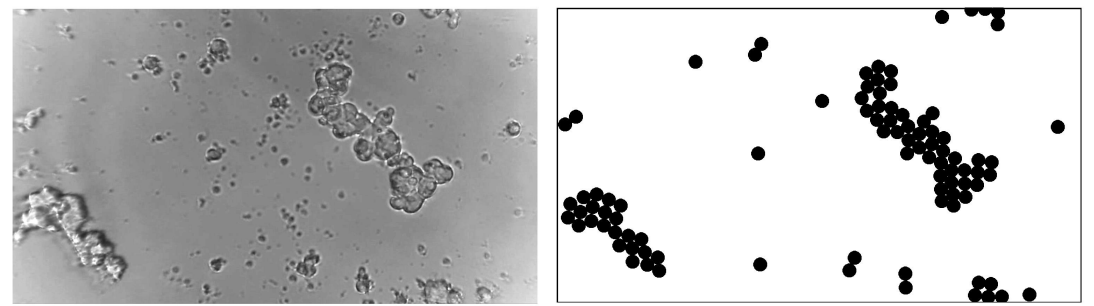

(c) $t=4$

Figure 1: Frames from a time-lapse video of the formations of clusters or islets of pancreatic cells (left), and the pattern of black circles that are used to represent the larger cells (right). Video frames courtesy The Brighton Centre for Regenerative Medicine.

developed here assumes that the cells experience a force which is proportional to the gradient of the concentration of a chemical signal in the medium which contains the cells. The standard laws of motion are then used to derive a system of differential equations which can be solved to give the locations of the cells and clusters at any required time.

Work concerning how chemical signals, such as morphogens, spread out from cells and how the gradient of these chemicals influence the formation of clusters and groups of cells has be reported in the literature. A review of the role that morphogens play in embryonic development is given in Lawrence and Struhl [14], which includes a discussion of morphogen gradients and how they work. Baker and Maini [2] developed a system of reaction-diffusion equations to model traveling waves of morphogens, and how the resulting gradients in the morphogen concentration can provide spatial information during embryonic development. Kornberg and Guha [12] discuss how the gradients of morphogens spread from regions of high concentration to regions of low concentration by various processes, including diffusion, and how this influences embryonic development. A 
more recent review of morphogenic gradients is given in Wolpert [21]. Although the diffusion and concentrations of morphogens are not of direct interest to this work, they do illustrate how chemical gradients in the surrounding medium can influence the behavior of cells.

In more recent work, the process of a cell emitting a chemical signal has been observed in experimental work concerned with cells secreting proteins, as reported in $[18,19]$. Assuming that the fluid in which the cells are immersed is essentially at rest, the spread of the chemical can be modelled using the linear diffusion equation. As the chemical spreads out from the emitting cell, there will be a gradient in its concentration levels that will attract the other clusters towards the emitting cell. The images in $[18,19]$ show that the proteins are spreading uniformly in all directions which is typical of the sort of spreading that can be modeled using the diffusion equation.

There are essentially two complimentary methods for modeling cell migration and clustering which can be broadly described as population based or individual based. Population based methods consider the population density of one or more cell types within an environment where they can react with chemical signals given out by other cells. In these models diffusion-reaction type equations are used to simulate how the population density of the cell evolves with time (see $[3,6,10,13]$ for example). Such models are often called Keller-Segel type models in the literature. The alternative is to use an individual based model where the individual cells (or clusters of cells) are each modelled separately. These models can range in complexity from those that assume that each cell can be represented by a simple geometric shape, (see $[5,11,20]$ for example) to mathematical models of how a single cell moves in response to it detecting chemical signal at its outer membrane (see [4] for example).

Eyiyurekli et al [5] propose a mathematical model for chemotaxis based cell aggregation based on each cell emitting a chemical signal that gives each cell a field or domain of influence which is determined by the chemical concentration being above a given threshold. If a second cell is within the field of influence then there is a mutual attraction between the two cells. The amount of signal chemical that is released by a cell is determined by a simple model of life-cycle of the cell. The magnitude of the chemical signal at a point outside the cell is expressed as a function of where the cell is in its life-cycle and the distance from the centre of the cell. However, the model does not include the diffusion of this chemical signal into the surrounding fluid and so the whole field of influence grows and decays as a single quantity. The velocity of the cells is assumed to be proportional to the chemical gradient meaning that the inertia terms are neglected in [5].

A one dimensional model developed by Thompson et al [20] considers how cells move across a lattice and derive a set of stochastic mean equations to determine how the cells move from one compartment in the lattice to an adjoining compartment. Whilst this model is based on cells moving from one compartment to another, it does not follow the motion of individual cells and simply gives the number of cells in each compartment at each time-step.

Kim et al [11] simulate the formation of clusters of cells in tumors and model the location of each individual cell within the cluster. The model simulates how a cell reacts to the levels of two types of cyclin-dependent kinases proteins and this is developed into a system of ordinary differential equations for quantities such as the cell's location and radius, and the levels of each protein within the cells. 
The paper also includes effects such as the life-cycle of the cells and different cell sizes. However, the cell's inertia is not included in the model described in [11] and equates the rate of change of the cells velocity to the applied forces. Further, although this model incorporates aspects of cells moving in response to chemical-like signals, it does not use a diffusion model for the spreading of the chemical signals.

A finite element type model of how an individual cell can move along a chemical signal and how that cell changes shape has been developed by Elliot et al [4]. The results presented in [4] illustrate how it is possible to simulate how a cell moves along a chemical signal by changing shape as its outer membrane reacts to the gradient in the chemical signal, and how cell changes direction when the direction in which the gradient is increasing changes. However, the computational cost of this type of model means that it is only feasible to consider problems with only a small number of cells.

An alternative finite element method using a mesh which moves with the cell it surrounds has been proposed by MacDonald et al [15] to model how a single cell moves due to chemotaxis. However, similar to the method proposed in [4], this method would be computationally expensive when considering a large number of cells. If the problem was started with each individual cell surrounded by its own finite element mesh, an method for combining the meshes when the cells become close together would need to be developed, or the whole domain around the cells would have to be meshed. There is also the question of what boundary condition to impose on the outer boundary of the finite element mesh.

In the model presented here, an exact expression for the concentration of the chemical signal emitted by a cell is used. This avoids the need for a computationally expensive numerical method for solving the diffusion equation over the domain of interest. It also avoids the need for specifying a boundary condition at the edge of the domain which is assumed to be infinite in extent. Further the gradient of the concentration can be obtained by differentiating the expression for the concentration with respect to each of the appropriate space variables in turn. However, the simplicity of the proposed model means that it is not possible to include any reaction terms where the chemical signal is modified by a cell when sensing the gradient of the signal.

The gradient of the chemical concentrations acts like an attractive force on the cells and as a result they experience the equivalent of a force acting in the direction of increasing concentration. Newton's second law of motion can be used to compute the acceleration of an object due to the forces applied to that object. In this work the attractive force on a cluster of cells will be determined by the gradient of the chemical signals released by other clusters in the vicinity of the cluster of interest. In addition frictional or drag forces, which are proportional to the objects velocity will be included in the model. The resulting system of differential equations for the cell (or cluster) locations and velocities are integrated through time using a adaptive fourth-order RungeKutta scheme which is more sophisticated than the Euler's methods which have been widely used in previous work. The Runge-Kutta method was chosen for this work as over a single time-step it is generally considerably more accurate than the Euler method, and is known to be more stable [1].

The model presented here can be used to solve either two or three dimensional problem. For brevity, only the model for two dimensional problems will be described in detail and the extension to three-dimensional problems will be 
given in outline.

\section{Mathematical Model For Two Dimensional Prob- lems}

Assume that the cells are immersed in a thin layer of fluid where the thickness of the fluid layer is approximately the same as the size of the cells, and that the layer is thin enough that the vertical variations in the concentration of the chemical can be neglected. The experimental results given in [17] show that the cells are approximately circular and do not change shapes as they move, and so it is also assumed that the cells can be considered as circles of radius $R$, and they are rigid in the sense that each cell cannot change shape as it moves. For computational convenience each cell is is considered to be contained in a cluster, although a cluster may consist of only a single cell. Let $C_{i}$ denote the $i^{\text {th }}$ cluster of cells and let $c_{i}$ denote the $i^{\text {th }}$ cell. The notation $c_{j} \in C_{i}$ is used to denote that the $j^{\text {th }}$ cell is part of the $i^{\text {th }}$ cluster. Two or more cells form a cluster if the distance between the centres of the two cells is less than or equal to twice the radius of the cells. Once two or more cells form a cluster, their relative locations within the cluster are assumed to be fixed and all cells in the cluster move together as a rigidly fixed group.

\subsection{Diffusion Of The Chemical Signal}

Let the centre of the $i^{\text {th }}$ cell be located at the point with coordinates $\left(x_{i}, y_{i}\right)$, which will change with time as the cell moves. At time $t=t_{i}$ (where $t_{i}$ is called the trigger time) the $i^{\text {th }}$ cell releases a chemical signal that attracts all the other cells. For simplicity, in the current model each cell will only emit a chemical signal at most one time during the simulation, and the time at which it releases the chemical is chosen randomly.

For problems with two space dimensions the chemical signal spreads out from the cell according to the linear diffusion equation

$$
\frac{\partial u_{i}}{\partial t}=\mu\left(\frac{\partial^{2} u_{i}}{\partial x^{2}}+\frac{\partial^{2} u_{i}}{\partial y^{2}}\right)
$$

where $u_{i}$ is the concentration of the signal chemical produced by the $i^{\text {th }}$ cell and $\mu>0$ is the diffusion constant. If each cell is treated as a point-source for the chemical signal, then it straightforward to show that

$$
u_{i}(x, y, t)= \begin{cases}\frac{A_{i}}{\mu\left(t-t_{i}+t_{\epsilon}\right)} \exp \left(-\frac{d(x, y)}{4 \mu\left(t-t_{i}+t_{\epsilon}\right)}\right) & t \geq t_{i} \\ 0 & t<t_{i}\end{cases}
$$

where

$$
d(x, y)=\left(x-\tilde{x}_{i}\right)^{2}+\left(y-\tilde{y}_{i}\right)^{2}
$$

is the solution to the diffusion equation (1). Here $A_{i}$ is the magnitude of the chemical signal produced by the $i^{\text {th }}$ cell, $t_{\epsilon}$ is a small value used to avoid problems with division by zero when $t=t_{i}$ (the value $t_{\epsilon}=0.01$ has been used for 
the two-dimensional results presented in this paper) and $\left(\tilde{x}_{i}, \tilde{y}_{i}\right)$ denotes the location of cell $i$ at time $t=t_{i}$ when it triggers its chemical signal. It is necessary to specify the cell location at the time that is triggers its chemical signal as the cell can still move after it has triggered but the chemical signal it produces will not move with it. The total concentration $u$ of the chemical at any point $(x, y)$ and at any time $t$ is simply the sum of the concentrations from each cell:

$$
u(x, y, t)=\sum_{i=1}^{N} u_{i}(x, y, t) .
$$

\subsection{The Equations Of Motion For The Cells}

It is assumed that the force acting on the cells is proportional to the gradient of the concentrations of the chemical at the centre of the cells. Every cell in each of the clusters now experience a force which is proportional to the gradient of the concentration of the chemical signal. If there are $n_{j}$ cells in the $j^{\text {th }}$ cluster then the total $x$ and $y$ components of the force acting on the cluster are given by

$$
\begin{aligned}
& F_{x j}=\sum_{j=1}^{n_{j}} \frac{\partial u\left(x_{j}, y_{j}, t\right)}{\partial x}=\sum_{j=1}^{n_{j}}\left(\sum_{i=1}^{N} \frac{\partial u_{i}\left(x_{j}, y_{j}, t\right)}{\partial x}\right) \\
& F_{y j}=\sum_{j=1}^{n_{j}} \frac{\partial u\left(x_{j}, y_{j}, t\right)}{\partial y}=\sum_{j=1}^{n_{j}}\left(\sum_{i=1}^{N} \frac{\partial u_{i}\left(x_{j}, y_{j}, t\right)}{\partial y}\right) .
\end{aligned}
$$

From Newtons Second Law, the $(x, y)$ components of the acceleration of the cells in the $j^{\text {th }}$ cluster are

$$
\begin{aligned}
& m_{j} \frac{d^{2} x_{j}}{d t^{2}}=F_{x j}-\lambda \frac{d x_{j}}{d t} \\
& m_{j} \frac{d^{2} y_{j}}{d t^{2}}=F_{y j}-\lambda \frac{d y_{j}}{d t}
\end{aligned}
$$

for all $c_{j} \in C_{j}$ where $m_{j}$ is mass of the cluster, the acceleration is expressed as the second time derivative of the location and $\lambda$ is a constant used to model the effect of the viscous damping force which is assumed to be proportional to the cluster's velocity. If all the cells under consideration have the same mass, say $m_{\text {cell }}$, then $m_{j}=m_{\text {cell }} \times n_{j}$. The above second order differential equations can be expressed as a system of first order equations in the form

$$
\begin{aligned}
& \frac{d v_{x j}}{d t}=\frac{1}{m_{j}}\left(F_{x j}-\lambda v_{x j}\right)=\frac{1}{m_{j}}\left(\sum_{j=1}^{n_{j}} \sum_{i=1}^{N} \frac{\partial u_{i}\left(x_{j}, y_{j}, t\right)}{\partial x}-\lambda v_{x j}\right) \\
& \frac{d v_{y j}}{d t}=\frac{1}{m_{j}}\left(F_{y j}-\lambda v_{y j}\right)=\frac{1}{m_{j}}\left(\sum_{j=1}^{n_{j}} \sum_{i=1}^{N} \frac{\partial u_{i}\left(x_{j}, y_{j}, t\right)}{\partial y}-\lambda v_{y j}\right) \\
& \left.\begin{array}{l}
\frac{d x_{k}}{d t}=v_{x j} \\
\frac{d y_{k}}{d t}=v_{y j}
\end{array}\right\} c_{k} \in C_{j}
\end{aligned}
$$


where $v_{x j}$ and $v_{y j}$ are the $(x, y)$ components of the velocity of all the cells in the $j^{\text {th }}$ cluster.

The initial conditions for this system of equations is that the location and velocity components are all known when $t=0$. In problem under consideration here, the initial velocity components will all be zero as the clusters are assumed to be stationary.

\subsection{Extension To Three-Dimensional Problems}

If the fluid layer is not thin compared to the size of the cells and/or if the vertical variations of the concentrations of the chemical cannot be neglected, then a three-dimensional model has to be used. In the three-dimensional problems considered in this paper, the cells are assumed to be rigid spheres of radius $R$.

If $z$ is used to denote the additional space dimension then the diffusion equation for the concentration of the chemical secreted by the $i^{\text {th }}$ cell becomes

$$
\frac{\partial u_{i}}{\partial t}=\mu\left(\frac{\partial^{2} u_{i}}{\partial x^{2}}+\frac{\partial^{2} u_{i}}{\partial y^{2}}+\frac{\partial^{2} u_{i}}{\partial z^{2}}\right)
$$

and the required point-source solution to this equation is

$$
u_{i}(x, y, z, t)= \begin{cases}\frac{A_{i}}{\mu\left(t-t_{i}+t_{\epsilon}\right)^{3 / 2}} \exp \left(-\frac{d(x, y, z)}{4 \mu\left(t-t_{i}+t_{\epsilon}\right)}\right) & t \geq t_{i} \\ 0 & t<t_{i}\end{cases}
$$

where

$$
d(x, y, z)=\left(x-\tilde{x}_{i}\right)^{2}+\left(y-\tilde{y}_{i}\right)^{2}+\left(z-\tilde{z}_{i}\right)^{2} .
$$

Note that (6) cannot be obtained from (2) by simply including the extra $z$ term in the function $d$ which appears in the exponential function as the power of the time-dependent term in the denominator is different.

The equations of motion for the cells in three-dimensions are a straightforward extension of those for two-dimensional problems by the inclusion of extra force and velocity equations for these quantities in the $z$ direction. That is, (5) will have two additional equations, one for $\frac{d v_{z j}}{d t}$ and one for $\frac{d z_{j}}{d t}$, which can be obtained from the corresponding equations for $x$ (or $y$ ) by replacing $x$ (or $y$ ) by $z$ in those equations.

\subsection{Time Integration Method}

It is virtually impossible to obtain a simple, closed-form analytical solution to system of differential equations given in (5). The alternative is to use an approximate or numerical method to solve the system. If $h$ denotes a timestep, then most of these numerical methods use the known solution at time $t$ to calculate the solution at time $t+h$.

There are a number of numerical methods for integrating one or more differential equations through time, and the details of these are given in most texts on numerical methods or numerical analysis (see [1] for example). The method that has been employed here is the fourth-order Runge-Kutta method. If $f(u, t)$ is a known function and $u_{n}$ and $u_{n+1}$ denote the approximate solutions of

$$
\frac{d u}{d t}=f(u, t)
$$


at times-steps $t_{n}$ and $t_{n+1}$ respectively, then

$$
u_{n+1}=u_{n}+\frac{h}{6}\left(k_{1}+2 k_{2}+2 k_{3}+k_{4}\right)
$$

where

$$
\begin{aligned}
& k_{1}=f\left(u_{n}, t_{n}\right) \\
& k_{2}=f\left(u_{n}+\frac{h}{2} k_{1}, t_{n}+\frac{h}{2}\right) \\
& k_{3}=f\left(u_{n}+\frac{h}{2} k_{2}, t_{n}+\frac{h}{2}\right) \\
& k_{4}=f\left(u_{n}+h k_{3}, t_{n}+h\right)
\end{aligned}
$$

and $h=t_{n+1}-t_{n}$ is the time-step (see [1], for example, for further details of this method).

The accuracy of the approximate solution will depend on the size of the time-step $h$. In order to control the accuracy of the numerical solution, a sequence time-steps $h_{1}>h_{2}>\cdots>h_{M}$ are used. The approximate solution at some later time is calculated with both time-steps $h_{1}$ and $h_{2}$, and if they are the same to within some pre-determined level of accuracy, the solution obtained using the smaller of the steps is taken as the required solution. If the solutions do not agree, then the next time-steps in the sequence are used until the solutions calculated using two consecutive time-steps agree to within the required accuracy. If this is not achieved after $M$ time-step divisions, then the method is deemed to have failed and the solution process is stopped. In the calculations reported here, $h_{k+1}=\frac{1}{2} h_{k}$ with $h_{1}=0.01$ and $M=15$. The time-step is also reduced if one or more cells move too far in a single time-step. Here the maximum permitted distance for a cell to move in a single time-step is $\frac{1}{10} R$. This is to ensure that the collisions between cells and clusters are accurately detected and to prevent two cells or clusters passing through each other without the collision being detected.

\subsection{Custer Collisions}

Two different clusters of cells are assumed to have collided if the distance between the centres of a cell in each cluster is less than twice the radius of a cell. That is, clusters $i$ and $j$ are assumed to have collided (in two-dimensions) if

$$
\min _{c_{i} \in C_{i}, c_{j} \in C_{j}}\left(\sqrt{\left(x_{i}-x_{j}\right)^{2}+\left(y_{i}-y_{j}\right)^{2}}\right) \leq 2 R .
$$

Once two clusters have collided they are combined to form a single, larger cluster. Further, assuming that all the cells have the same mass then for twodimensional problems the conservation of momentum requires that the velocity components $\left(v_{x}, v_{y}\right)$ of the new cluster after the collision are related to the velocity components $\left(v_{x i}, v_{y i}\right)$ and $\left(v_{x j}, v_{y j}\right)$ of the original clusters by

$$
v_{x}=\frac{n_{i} v_{x i}+n_{j} v_{x j}}{n_{i}+n_{j}} ; \quad v_{y}=\frac{n_{i} v_{y i}+n_{j} v_{y j}}{n_{i}+n_{j}} .
$$

In the three-dimensional case, there will be an extra $\left(z_{i}-z_{j}\right)^{2}$ term inside the square-root function in (7) and an extra equation for $v_{z}$ in (8). 


\section{$3 \quad$ Numerical Results}

The results presented here are scaled such that the cells have radius one unit $(R=1)$ and mass one unit $\left(m_{\text {cell }}=1\right)$. For the two-dimensional problems considered here, the initial clusters are randomly located with between 1 and 4 cells in each cluster, and each cell is assigned a random trigger time. The results presented below are for the initial clusters shown in Figure 2 where there is 123 cells arranged in 50 clusters. The size of the square box surrounding the clusters is 106 times the radius of a single cell.

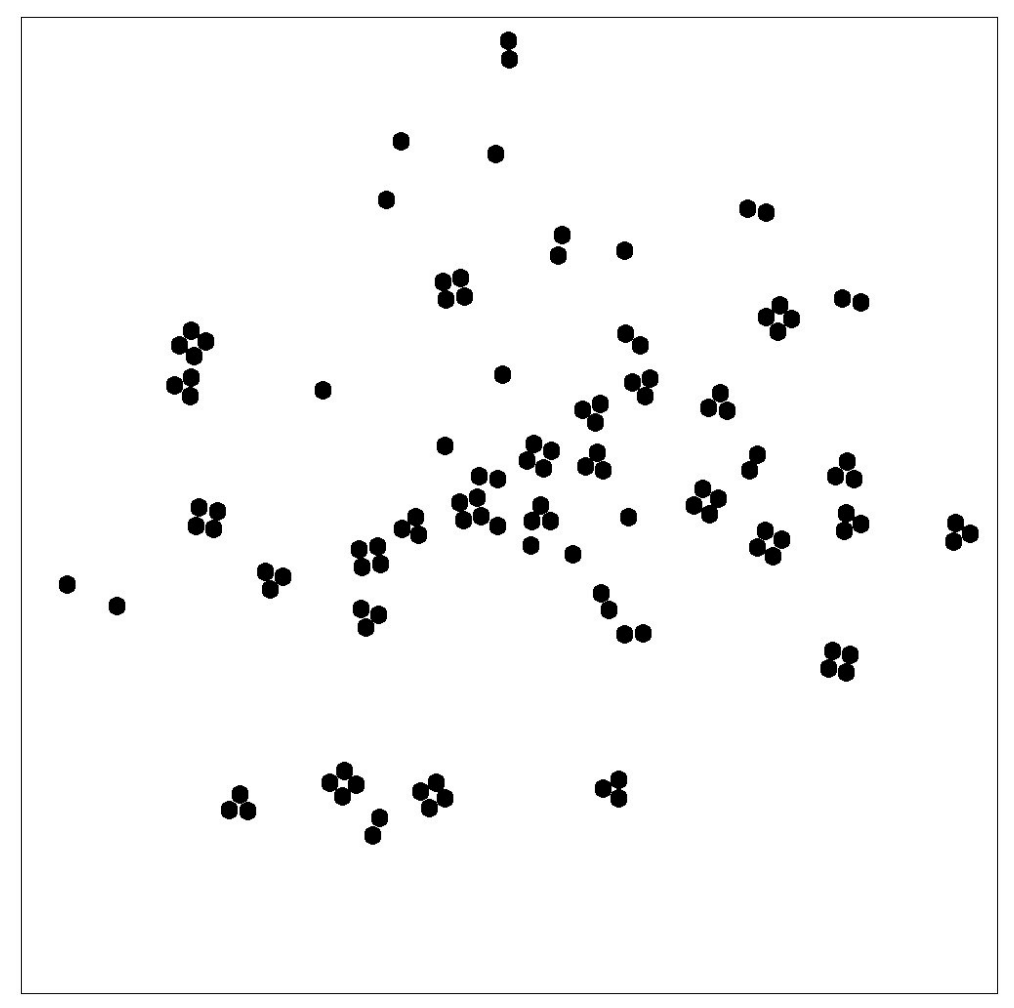

Figure 2: The initial locations of the 50 clusters of cells.

Figure 3 shows the results at time $t=500$ using four different chemical concentration parameters $A_{i}$ (see equation (2)) with the diffusion constant $\mu=$ 1 and damping coefficient $\lambda=500$. These results show that increasing the concentration parameter increases the rate at which the cells cluster together. The cell distribution shown in Figure 3(a), which used the smallest value of $A_{i}$, shows that there has been relative little movement of the cells and clusters away from the initial distribution shown in Figure 2, whereas Figure 3(d) shows that most of the clusters have moved relatively large distances and collided to form a single large cluster with only a small number of outlying smaller clusters. This is as expected as increasing the parameter $A$ simply increases the size of the concentration and its gradient and so effectively applies a greater attractive force to the cells and clusters. 


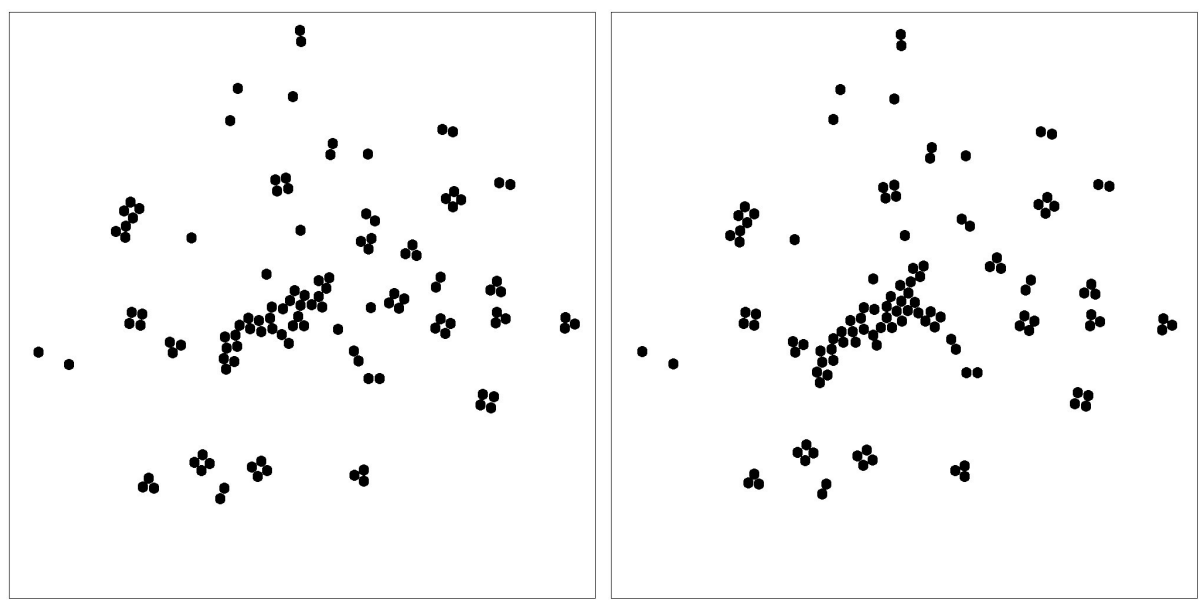

(a) $A_{i}=987.5$

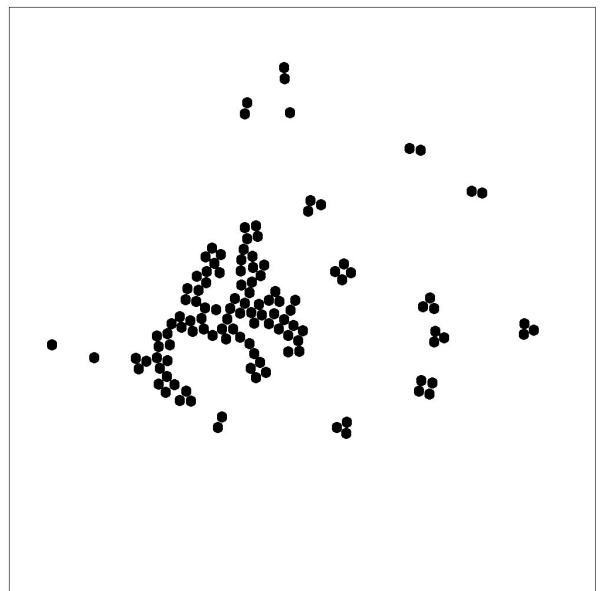

(c) $A_{i}=9875$ (b) $A_{i}=1975$

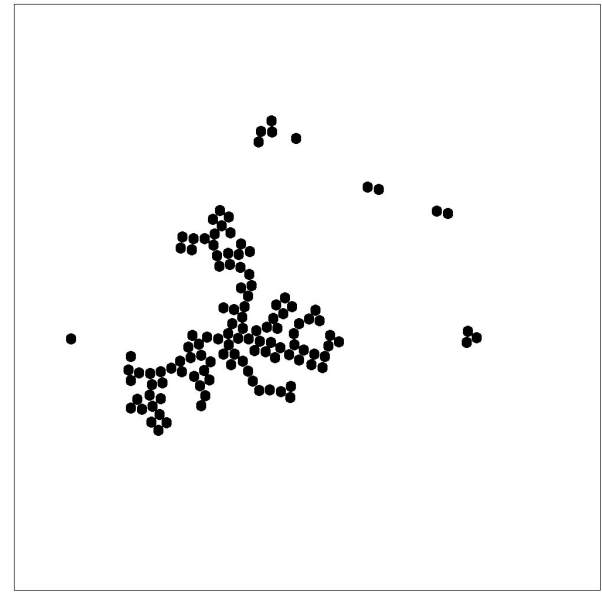

(d) $A_{i}=19750$

Figure 3: The locations of the cells after 500 units of time using different values of the concentration parameter $A_{i}$ in equation (2).

Figure 4 shows the results at time $t=500$ of changing the diffusion parameter $\mu$, with $A_{i}=9875$ and $\lambda=500$. Increasing the value of the diffusion constant reduces the rate at which the cells cluster together. This is because as $\mu$ increases the rate at which the chemical concentration spreads out also increases, meaning that it quickly reaches a state where the magnitude of the gradient is small. Consequently, the concentration is only applying a small force to the cells. However for smaller values of $\mu$ the chemical concentration spreads much slower meaning that there are steep concentration gradients for more time which in turn apply greater forces to the cells. Hence in the cells in Figure 4(a) have collected together into large clusters because of the larger concentration gradients that occur for $\mu=0.2$ whereas Figure $4(\mathrm{~d})$ shows less clustering as $\mu=10$ in this case.

The results of changing the damping force, controlled using the parameter 


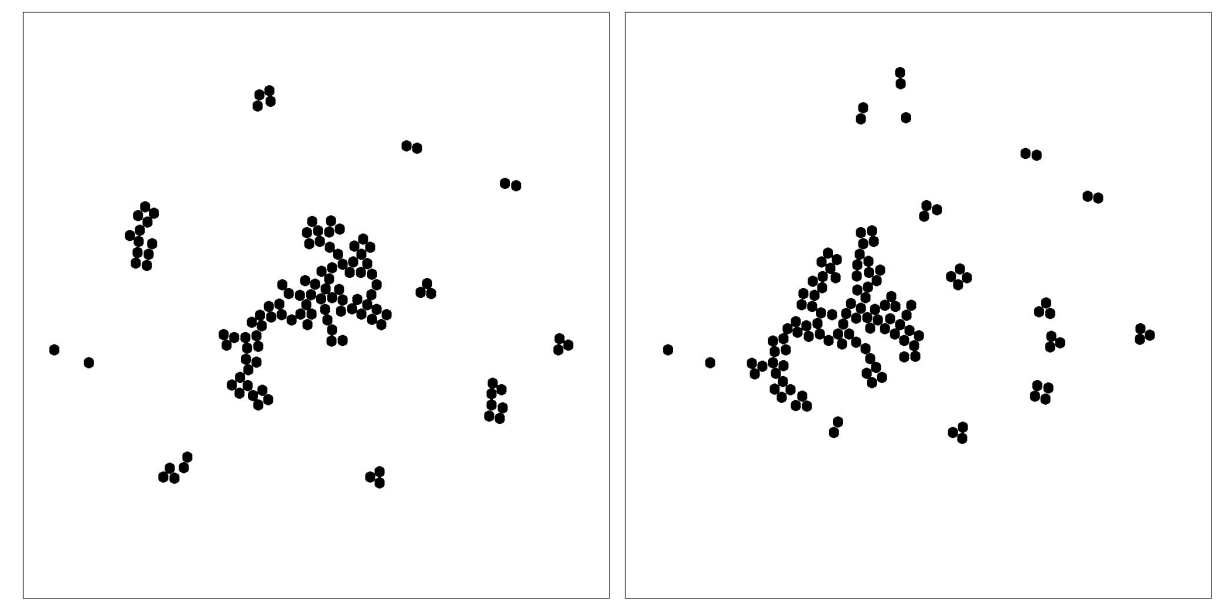

(a) $\mu=0.2$

(b) $\mu=1.0$

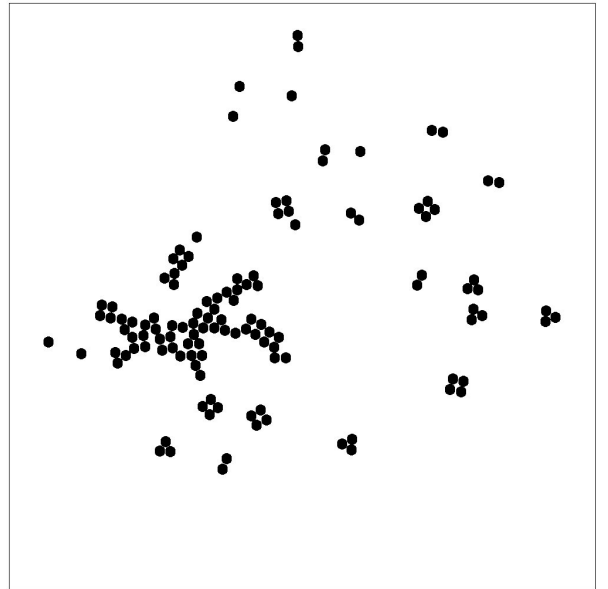

(c) $\mu=5.0$

(d) $\mu=10.0$

Figure 4: The locations of the cells after 500 units of time using different values of the diffusion constant $\mu$ in equation (2).

$\lambda$ in (4), at time $t=500$ are shown in Figure 5 . Here the values $A_{i}=9875$ and $\mu=1$ have been used. In Figure 5 (a) $\lambda$ is relatively small and so the motion of the cells and clusters is not impeded. However, as $\lambda$ increases, as shown in Figures $5(\mathrm{~b})$ to $5(\mathrm{~d})$ the effect of the damping force is enough to prevent the cells and clusters combining to form larger clusters. Again, this is as expected since increasing the damping on the cells will reduce the rate at which they accelerate.

The calculated locations of the cells for a second example with 1000 initial clusters containing 2443 cells at different times is shown in Figure 6. In this example the values $A_{i}=19875, \mu=1$ and $\lambda=500$, and the cells are initially contained in a square box with sides of length 400 times the radius of a single cell. A video of this example is available with the online supplementary material. This example shows how the model developed in the paper can be used to 


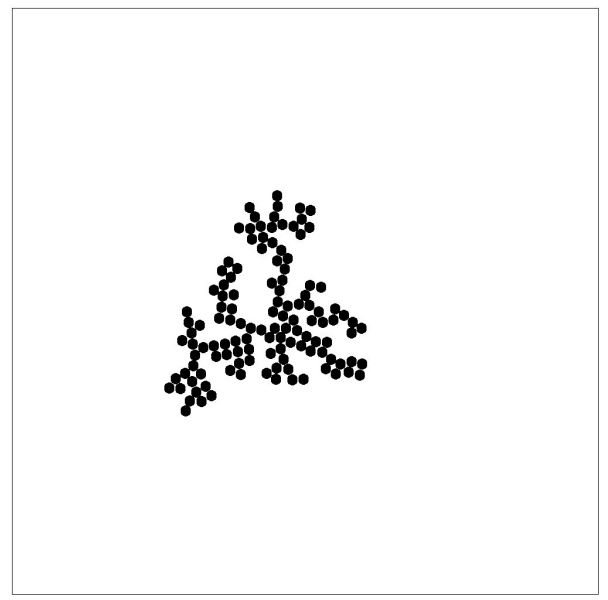

(a) $\lambda=50$

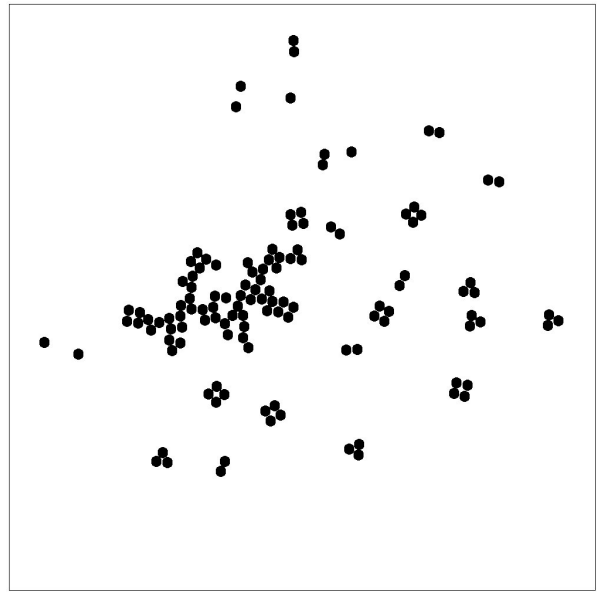

(c) $\lambda=1000$

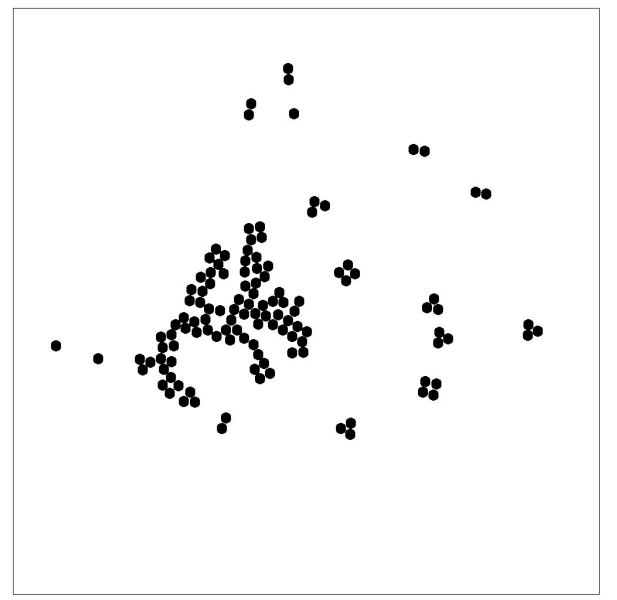

(b) $\lambda=500$

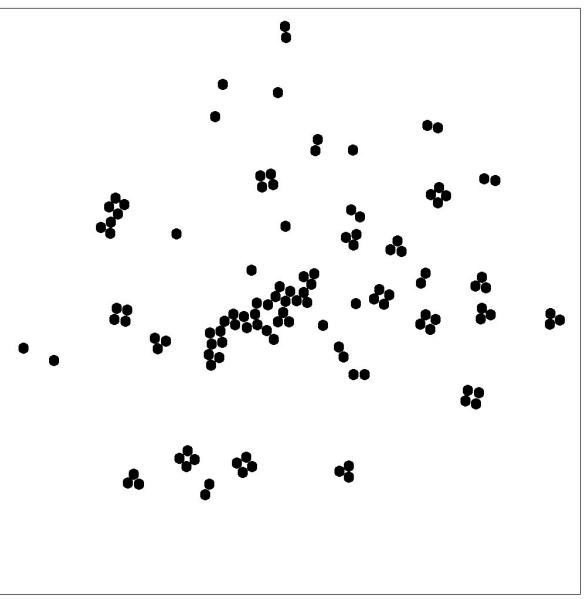

(d) $\lambda=5000$

Figure 5: The locations of the cells after 500 units of time using different values of the drag/damping constant $\lambda$ in equation (4).

simulate how relatively large numbers of scattered cells can signal to each other and come together to form large clusters of cells.

Figure 7 show the results of simulating a three-dimensional problem with 100 isolated cells. For this example $A_{i}=98750, \mu=1$ and $\lambda=500$. In this case, $t_{\varepsilon}=0.1$ as the higher power of the term involving $t$ in the denominator of (6) can lead to computational problems when $t-t_{i}$ is small. The bounding box containing the cells is a cube with sides whose lengths are 52 times the radius of a cell. A video of this example is available in the online supplementary material. The results from this example show that the method can be used to simulate the clustering of cells in three-dimensional problems as well as two-dimensional ones. 


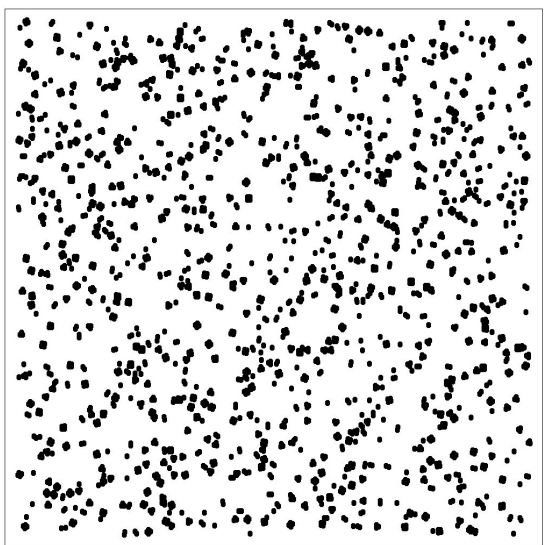

(a) $t=0$

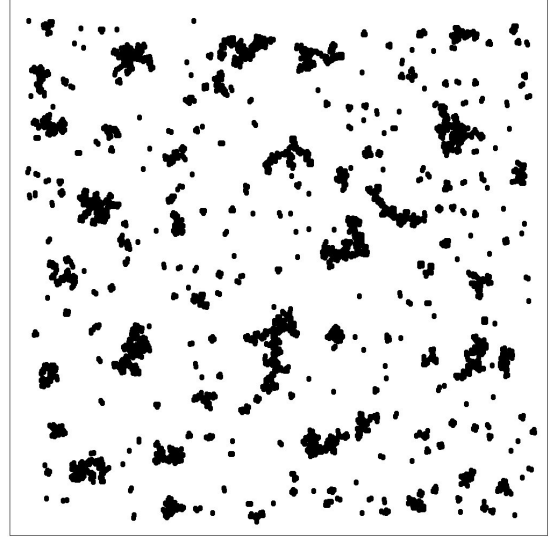

(c) $t=400$

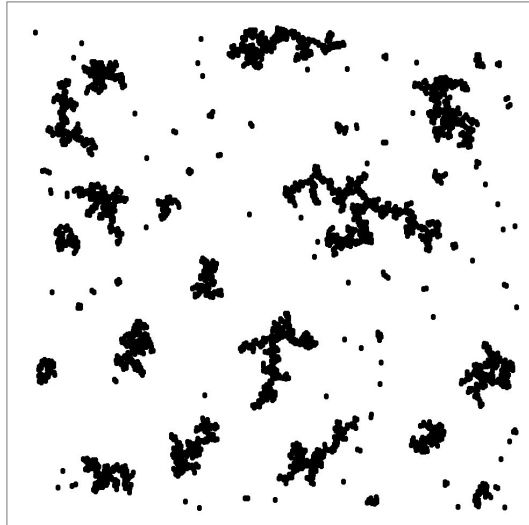

(e) $t=800$

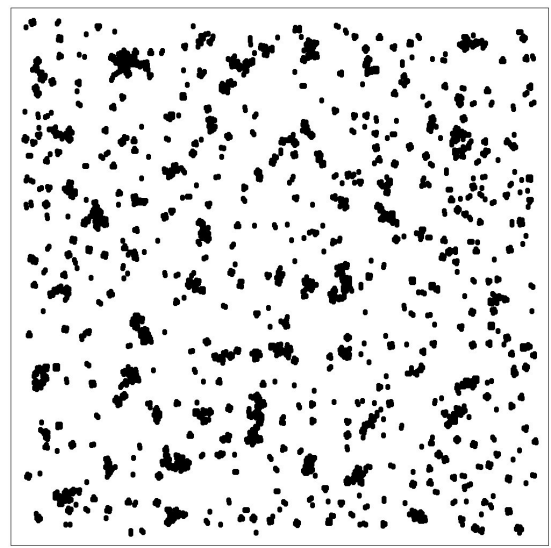

(b) $t=200$

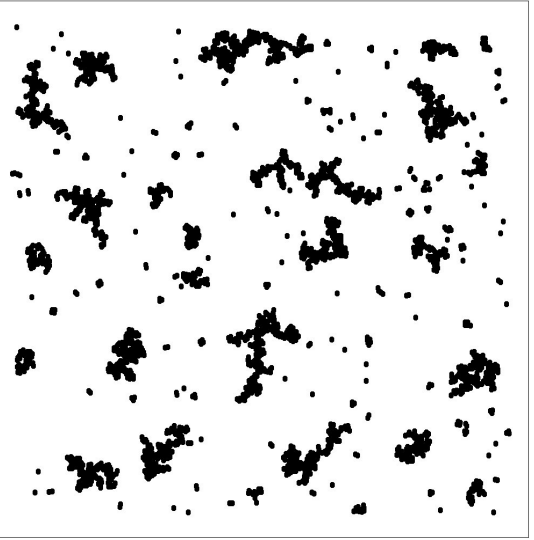

(d) $t=600$

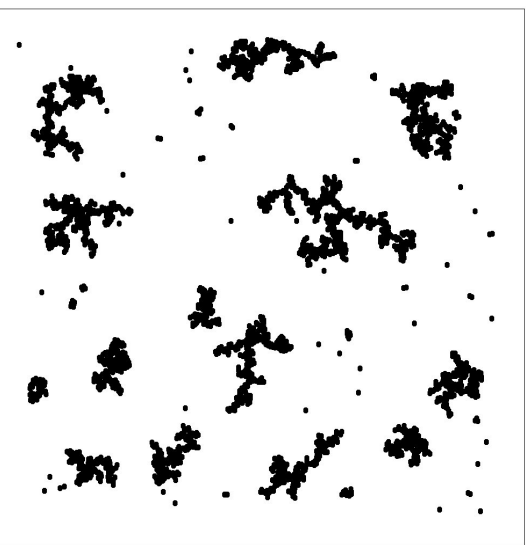

(f) $t=1000$

Figure 6: The locations of the cells at different times for an example with 2443 cells in 1000 initial small clusters.

\section{Conclusions}

The numerical results presented in Section 3 of this paper demonstrate that this model is effective at modeling how cells cluster together due to chemotaxis. 


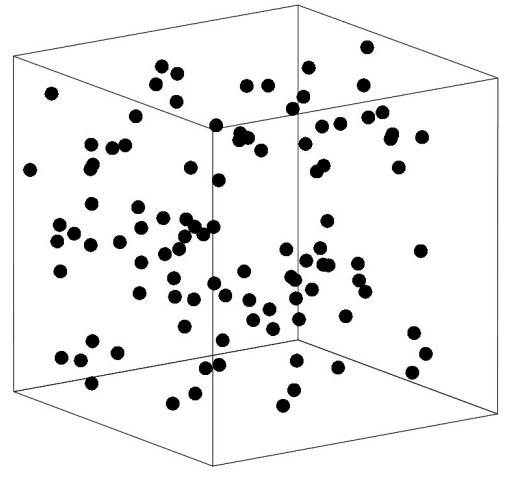

(a) $t=0$

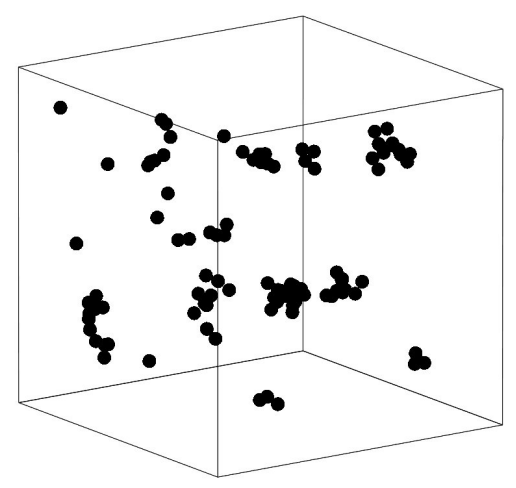

(c) $t=80$

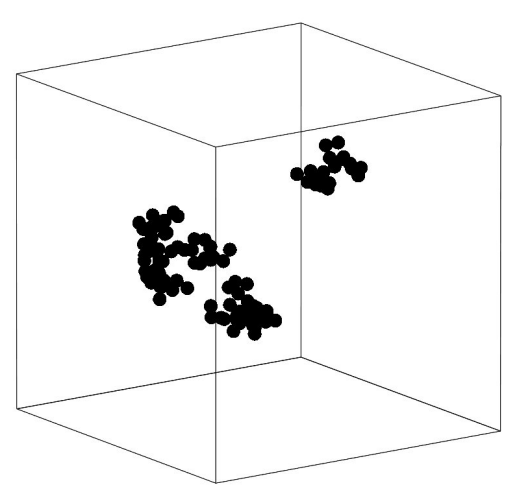

(e) $t=160$

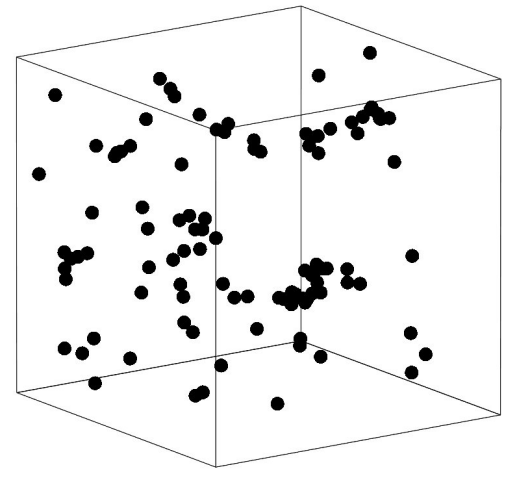

(b) $t=40$

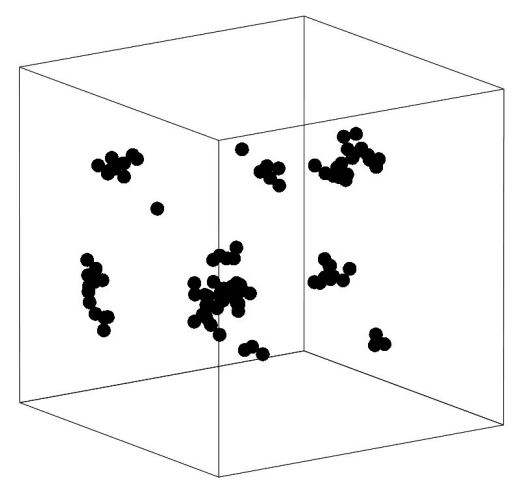

(d) $t=120$

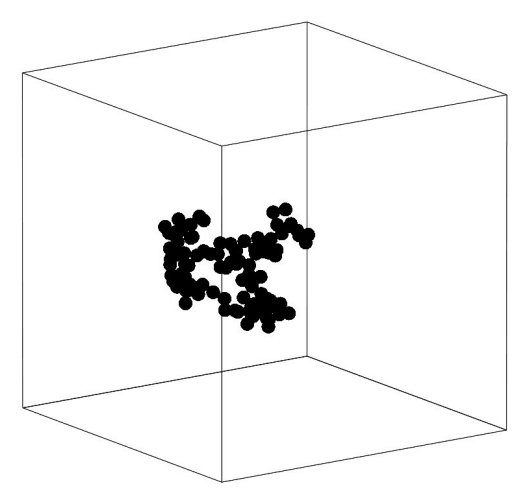

(f) $t=200$

Figure 7: The locations of the cells at different times for a three-dimensional example with 100 cells. 
The rate at which the cells and clusters combine can be controlled by choosing appropriate values of the chemical concentration magnitude $\left(A_{i}\right)$, diffusion constant $(\mu)$ and damping coefficient $(\lambda)$. Increasing the concentration magnitude increases the speed at which the cells and clusters combine into large clusters, and increasing the damping coefficient $\lambda$ slows the process down, as expected. Increasing the diffusion constant $\mu$ causes the chemical to spread out more rapidly, and this leads to a reduction in the size of the concentration gradient. This, in turn, reduced the force acting on the cells and clusters and so inhibits the cells and clusters combining together. Further experimental work is needed to determine the appropriate values of these parameters for particular cells as the values of these parameters are likely to change for different types of cells.

Although using the different time-steps to control the accuracy adds to the computational cost of the model, this cost is not excessive as the simulations using 123 cells in 50 clusters typically needed approximately 1.5 minutes of CPU time to run on a typical desk-top PC. Hence the methods presented in this paper are an efficient way of accurately simulating how cells and clusters of cells move and combine due to chemotaxis. The larger example, with 2443 cells in 1000 clusters, needed approximately 25 hours and 14 minutes of CPU time to run on the same PC. The rapid increase in CPU time is to be expected, as the number of pair-wise interaction between cells (and clusters) increases as approximately in proportion to the number of cells (or clusters) squared. The three-dimensional example with 100 cells required approximately 21.5 minutes of CPU time on the same PC, but this time includes the added complexity of dealing with both the extra space dimension and the stronger time discontinuity in (6) which means that the CPU time for three-dimensional examples cannot be directly compared to the CPU time for the two-dimensional problems.

The amount of CPU time required for the model presented here can be compared to that required for the more sophisticated finite element models presented in [4] and [15]. Elliott et al [4] report that the calculations for the results for modeling the motion of five cells presented in Figure 3 of [4] required approximately 33 minutes of CPU time, whilst McDonald et al [15] report that their finite element method typically took 3 hours of CPU time to model to motion of a single cell. Scaling either of these models up to examples with thousands of cells is not feasible due to the very high computational cost involved. In addition the model presented here used an adaptive time-stepping scheme to control the accuracy of the numerical results. The use of such time-stepping schemes would increase the computational cost of the finite element method significantly, although it is noted that the authors of $[4,15]$ do discuss the temporal convergence of their respective methods.

The model presented here can be extended in a number of ways. Firstly, once two or more clusters have combined the location of the cells remain fixed relative to other cells in the cluster and the whole cluster essentially moves as a rigid body. This can lead to clusters forming strange shapes (see Figure 6(f) for example). To overcome this, a mechanism for cells to move within a cluster needs to be incorporated into the model. Secondly, this model does not consider the life-cycle of the cells, or whether cells can divide. Methods described in both [11] and [5] could be used to model each cells life-cycle. Finally, the model does not include any reaction terms whereby the magnitude of the chemical signal is modified by a cell sensing the gradient. However, it likely 
that such effects cannot be included analytically meaning that the resulting reaction-diffusion equation would need to be solved numerically which would add considerably to the computational cost.

Acknowledgment

The author would like to thank Matteo Santin and Gary Phillips from The Brighton Centre for Regenerative Medicine for their help and advice with some of the biological aspects of this paper. 


\section{References}

[1] K.E. Atkinson. An introduction to numerical analysis. John Wiley and Sons, New York, 2 edition, 1989.

[2] R.E. Baker and P.K. Maini. Travelling gradients in interacting morphogen systems. Mathematical Biosciences, 209(1):30-50, SEP 2007.

[3] A. Chertock, A. Kurganov, X. Wang, and Y. Wu. On a chemotaxis model with saturated chemotactic flux. Kin. and Rel. Mod, 5(1):51 - 95, 2012.

[4] C.M. Elliott, B. Stinner, and C. Venkataraman. Modelling cell motility and chemotaxis with evolving surface finite elements. J. R. Soc. Interface, 9:3027 - 3044, 2012.

[5] M. Eyiyurekli, P. Manley, P.I. Lelkes, and D.E. Breen. A computational model of chemotaxis-based cell aggregation. BioSysytems, 93:226 - 239, 2008.

[6] H. Gajewski and K. Zacharias. Global behaviour of a reaction - diffusion system modelling chemotaxis. Math. Nachr, 195:77 - 114, 1998.

[7] J. Hilderink, S. Spijker, F. Carlotti, L. Lange, M. Engelse, C. van Blitterswijk, E. de Koning, M. Karperien, and A. van Apeldoorn. Controlled aggregation of primary human pancreatic islet cells leads to glucose-responsive pseudoislets comparable to native islets. Journal Of Cellular And Molecular Medicine, 19(8):1836-1846, AUG 2015.

[8] O. Hoeller and R.R. Kay. Chemotaxis in the absence of pip3 gradients. Current Biology, 17(9):813 - 817, 2007.

[9] D. Jia, D. Dajusta, and R.A. Foty. Tissue surface tensions guide in vitro self-assembly of rodent pancreatic islet cells. Developmental Dynamics, 236(8):2039-2049, AUG 2007.

[10] E.F. Keller and L.A. Segel. Model for chemotaxis. J. Theor. Biol., 30:225 $-234,1971$

[11] M. Kim, D. Reed, and K.A. Rejniak. The formation of tight tumor clusters affects the efficacy of cell cycle inhibitors: A hybrid model study. J. Theor. Biol., 352:31 - 50, 2014.

[12] T.B. Kornberg and A. Guha. Understanding morphogen gradients: a problem of dispersion and containment. Current Opinion in Genetics 83 Development, 17(4):264-271, AUG 2007.

[13] I.R. Lapidus and R. Schiller. A mathematical model for bacterial chemotaxis. Biophys. J., 14:825-834, 1974.

[14] P.A. Lawrence and G. Struhl. Morphogens, compartments, and pattern: Lessons from drosophila? Cell, 85:951 - 961, 1996.

[15] G. MacDonald, J.A. Mackenzie, M. Nolan, and R.H. Insall. A computational method for the coupled solution of reaction-diffusion equations on evolving domains and manifolds: Application to a model of cell migration and chemotaxis. J. Comp. Phys., 309:207-226, 2016. 
[16] S.E. Malawista, A.D. Chevance, and L.A. Boxer. Random locomotion and chemotaxis of human blood polymorphonuclear leukocytes from a patient with Leukocyte Adhesion Deficiency-1: Normal displacement in close quarters via chimneying. Cell Motility and the Cytoskeleton, 46(3):183-189, 2000 .

[17] N. Nitta, T. Tsuchiya, A. Yamauchi, T. Tamatani, and S. Kanegasaki. Quantitative analysis of eosinophil chemotaxis tracked using a novel optical device taxiscan. Journal of Immunological Methods, 320(12):155 - 163, 2007.

[18] M.P. Raphael, Joseph A. Christodoulides, J.B. Delehanty, J.P. Long, and J.M. Byers. Quantitative Imaging of Protein Secretions from Single Cells in Real Time. Biophysical Journal, 105(3):602-608, AUG 62013.

[19] Y. Shirasaki, M. Yamagishi, N. Suzuki, K. Izawa, A. Nakahara, J. Mizuno, S. Shoji, T. Heike, Y. Harada, R. Nishikomori, and O. Ohara. Real-time single-cell imaging of protein secretion. Scientific Reports, 4, APR 222014.

[20] R.N. Thompson, C.A. Yates, and R.E. Baker. Modelling cell migration and adhesion during development. Bull. Math. Biol., 74:2793 - 2809, 2012.

[21] L. Wolpert. Positional information and patterning revisited. Journal Of Theoretical Biology, 269(1):359-365, JAN 212011. 